\title{
Functional development of the octenol response in Aedes aegypti
}

\author{
Jonathan D. Bohbot ${ }^{1}$, Nicolas F. Durand ${ }^{1+}{ }^{,}$, Bryan T. Vinyard ${ }^{2}$ and Joseph C. Dickens ${ }^{1 *}$ \\ Invasive Insect Biocontrol and Behavior Laboratory, Henry A. Wallace Beltsville Agricultural Research Center, Plant Sciences Institute, United States Department of \\ Agriculture, Agricultural Research Service, Beltsville, MD, USA \\ ${ }^{2}$ Biometrical Counseling Service, Henry A. Wallace Beltsville Agricultural Research Center, United States Department of Agriculture, Agricultural Research Service, \\ Beltsville, MD, USA
}

\section{Edited by:}

Catherine Loudon, University of

California at Irvine, USA

Reviewed by:

Hong Lei, University of Arizona, USA

Ulrich Theopold, Stockholm

University, Sweden

*Correspondence:

Joseph C. Dickens, Invasive Insect Biocontrol and Behavior Laboratory,

Beltsville Agricultural Research

Center, Plant Sciences Institute,

United States Department of

Agriculture, Agricultural Research

Service, Building 007, Room 030,

10300 Baltimore Avenue, Beltsville, MD 20705, USA.

e-mail: joseph.dickens@ars.usda.gov

\section{${ }^{\dagger}$ Present address:}

Nicolas F. Durand, Physiologie de

I'Insecte, Signalisation et

Communication, Université Pierre et

Marie Curie, UMR 1272, F-75252

Paris, France.
Attraction of female Aedes aegypti mosquitoes to 1-octen-3-ol (octenol), $\mathrm{CO}_{2}$, lactic acid, or ammonia emitted by vertebrate hosts is not only contingent on the presence of odorants in the environment, but is also influenced by the insect's physiological state. For anautogenous mosquito species, like $A$. aegypti, newly emerged adult females neither respond to host odors nor engage in blood-feeding; the bases for these behaviors are poorly understood. Here we investigated detection of two components of an attractant blend emitted by vertebrate hosts, octenol, and $\mathrm{CO}_{2}$, by female $A$. aegypti mosquitoes using electrophysiological, behavioral, and molecular approaches. An increase in sensitivity of octenol olfactory receptor neurons (ORNs) was correlated with an increase in odorant receptor gene (Or) expression and octenol-mediated attractive behavior from day 1 to day 6 post-emergence. While the sensitivity of octenol ORNs was maintained through day 10, behavioral responses to octenol decreased as did the ability of females to discriminate between octenol and octenol $+\mathrm{CO}_{2}$. Our results show differing age-related roles for the peripheral receptors for octenol and higher order neural processing in the behavior of female mosquitoes.

Keywords: odorant receptor, gustatory receptor, octenol, $\mathrm{CO}_{2}$, olfaction, Aedes aegypti, development

\section{INTRODUCTION}

Female Aedes aegypti mosquitoes spread human pathogenic viruses that cause yellow fever, dengue fever, and Chikungunya. Much of the struggle against these diseases has relied on a combination of prophylactic measures such as vector control including insecticides and odor-baited traps. These systems can be improved once we better understand the relationships between the A. aegypti olfactory system and odorants.

The olfactory system of insects is divided into olfactory receptor neurons (ORNs), which constitute the peripheral olfactory system, and higher brain centers which receive messages from the ORNs (Galizia and Rossler, 2010). Sensilla distributed on the antennae, maxillary palps, and proboscis of mosquitoes (Figure 1A) house ORNs that detect volatile chemicals and transduce these signals into electrical outputs for further processing within the central nervous system. Detection of specific odorants, such as $\mathrm{CO}_{2}$, octenol, lactic acid, and ammonia, is carried out by receptor proteins located in the dendritic membrane of ORNs; these receptors belong to the odorant receptor $(\mathrm{OR})$, gustatory receptor (GR), and ionotropic receptor classes (Hansson and Stensmyr, 2011).

Maxillary palps (Figure 1A) of female A. aegypti harbor 2935 club-shaped basiconic sensilla (McIver, 1982; Figure 1B) that house specific ORNs that detect the attractants octenol and $\mathrm{CO}_{2}$
(Takken and Kline, 1989; Grant and O'Connell, 1996). These multiporous basiconic sensilla (Figure 1C) are the only chemosensory organs found on the maxillary palps and are innervated by three ORNs (Figure 1D), each generating a characteristic action potential. The largest amplitude action potential from the "A" neuron responds to fluctuations of $\mathrm{CO}_{2}$ (Grant and O'Connell, 1996). A cognate stimulus is unknown for the " $\mathrm{B}$ " neuron characterized by an intermediate amplitude spike (Figure $2 \mathrm{~A}$ ). The "C" neuron, which produces the smallest amplitude spike (Figure 2A) responds to the $R$-enantiomer of octenol (Grant and Dickens, 2011).

Studies in Anopheles gambiae have shown that the "C" neuron co-expresses the A. gambiae GR 22 ( $A g G r 22), A g G r 23$, and $A g G r 24$ genes (Jones et al., 2007; Lu et al., 2007), the orthologs of the Drosophila melanogaster $\mathrm{CO}_{2}$ receptors Gr21a and Gr63a (Jones et al., 2005; Kent et al., 2008; Robertson and Kent, 2009). Three orthologs have been identified in the maxillary palps of $A$. aegypti: $A a G r 1, A a G r 2$, and $A a G r 3$ (Kent et al., 2008; Robertson and Kent, 2009). While both $\mathrm{AaGrl}$ and $\mathrm{AaGr} 3$ have been implicated in $\mathrm{CO}_{2}$ sensing, the role of $A a G r 2$ is unknown (Erdelyan et al., 2011). The receptor assemblage comprised of the odorant sensing subunit AaOR8 and the obligatory coreceptor AaOrco specifically recognizes $(R)$-octenol (Bohbot and Dickens, 2009) and provides enantioselectivity to the "C" cell (Grant and Dickens, 2011). 

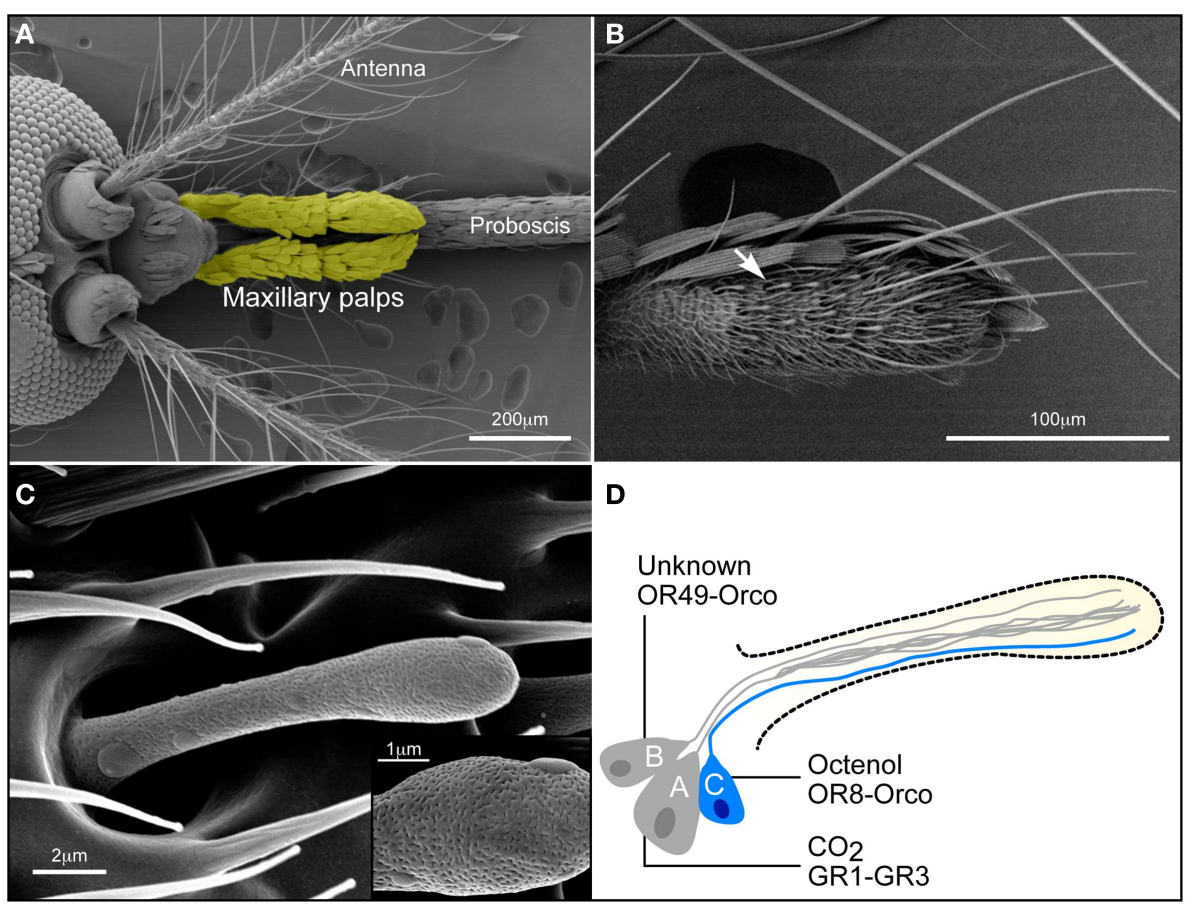

\section{FIGURE 1 | Olfactory system of the female Aedes aegypti} mosquito. (A) The $A$. aegypti head (dorsal view) possesses three olfactory appendages: the antennae, the maxillary palps (here colored yellow using Adobe Photoshop), and the proboscis. (B) Basiconic sensilla (white arrow) are the only chemosensory organs on the fourth segment of the maxillary palps (ventro-lateral view). (C) A basiconic sensillum is a club-shaped sensory organ. Pores (see insert) allowing odorants to penetrate the sensillum are densely distributed over the surface of the hair. (D) Diagram outlining the conceptual neuronal content of a basiconic sensillum. Three chemosensory neurons are enclosed within the sensillum. The $\mathrm{CO}_{2}$ or " $\mathrm{A}$ " neuron expresses AaGr1 and AaGr3. The "B" neuron is presumed to express AaOr49-Orco (unknown ligand). The AaOR8-Orco complex confers octenol sensitivity to the " $\mathrm{C}$ " neuron (labeled in blue).
While there is no direct evidence of $A a O r 8$ expression in the dendritic segment of the "C" cell, the A. gambiae Or8 ortholog is present in one of the three ORNs in the basiconic sensilla and AaOr8 is expressed in the maxillary palp (Bohbot et al., 2007).

Since only three Ors have been identified in the maxillary palps (Bohbot et al., 2007), the current model assumes that AaGrl, 2, 3 are expressed in the "A" neuron while AaOr49-Orco and AaOr8-Orco are expressed in the " $\mathrm{B}$ " and " $\mathrm{C}$ " neurons, respectively (Figure 1D).

For holometabolous insects, like mosquitoes, the adult phenotype is acquired during the metamorphic pupal stage. The adult peripheral sensory apparatus develops from imaginal disks and the brain undergoes dramatic remodeling. These profound changes accompany the organism's transition from an aquatic to a terrestrial life style, and ends shortly after emergence (Clements, 1999). In their first $24 \mathrm{~h}$ as imago, female mosquitoes do not blood-feed and their sensory capability is incomplete. For example, the physiological development of $\mathrm{CO}_{2}$-sensitive neurons on the palps of newly emerged female $A$. aegypti is age-dependent (Grant and O'Connell, 2007), much like the lactic-acid sensitive neuron on the antennae (Davis, 1984). It is unknown whether the physiology of the octenol-sensitive "C" neuron follows a similar developmental pattern in newly emerged and older adults.
In this study, we have focused our attention on the developmental aspects of octenol detection in adult female A. aegypti mosquitoes using molecular, cellular, and behavioral approaches. We show that genetic and physiological components directly involved in octenol detection are maturing $24 \mathrm{~h}$ post-emergence. Individual 1 day old mosquitoes show no chemotaxis toward either $\mathrm{CO}_{2}$ or $\mathrm{CO}_{2}+$ octenol while older animals are attracted by both. Our data indicate that the peripheral sensory system responsible for octenol detection is not mature at emergence, and suggest that associated behavioral responses to $\mathrm{CO}_{2}$ and $\mathrm{CO}_{2}+$ octenol are under temporal control by the peripheral and central olfactory systems.

\section{MATERIALS AND METHODS INSECTS}

Eggs of the Orlando (1952 Florida) strain of A. aegypti (L.) were obtained from The Center for Medical and Veterinary Entomology, USDA, ARS in Gainesville, FL, USA. Mosquitoes were reared in an environmental chamber at $27^{\circ} \mathrm{C}$ and $70 \%$ relative humidity with a 12:12 light-dark cycle. Larvae were fed on a fish food diet (Tetramin $\left.{ }^{\circledR}\right)$. Unsexed pupae were hand-collected and placed into small cages $(9 \mathrm{~cm} \times 5.5 \mathrm{~cm})$. Adults consistently emerged 2 days following pupation and were segregated according to their emergence date. Male and female adult mosquitoes were allowed to mate and were fed on a $10 \%$ sucrose solution ad libitum. 


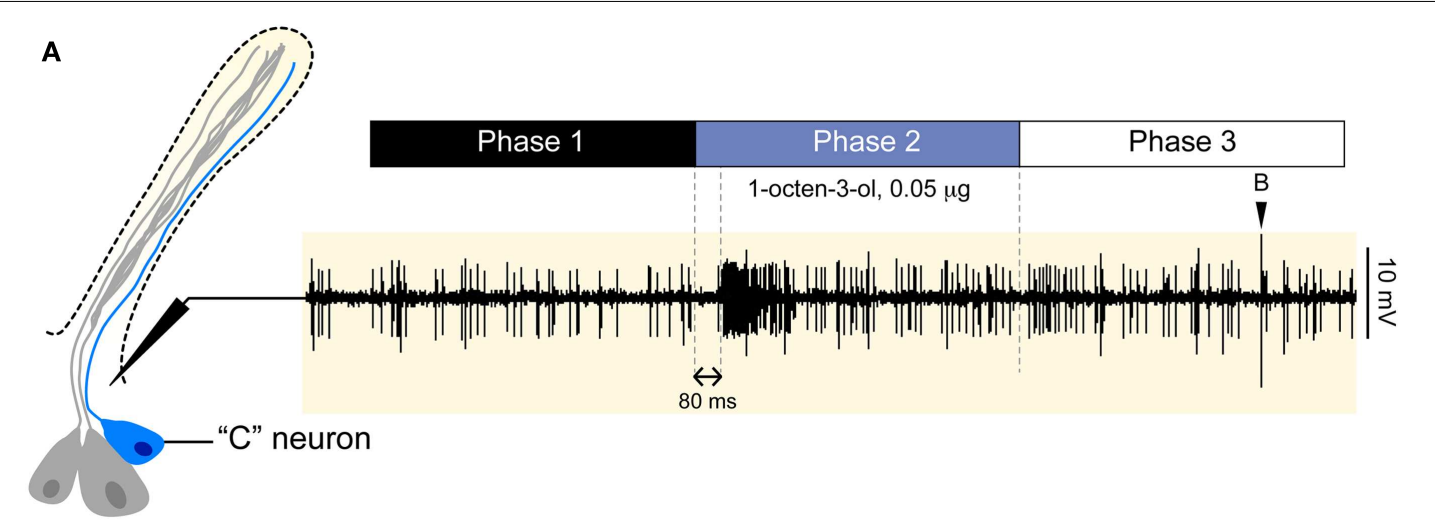

B

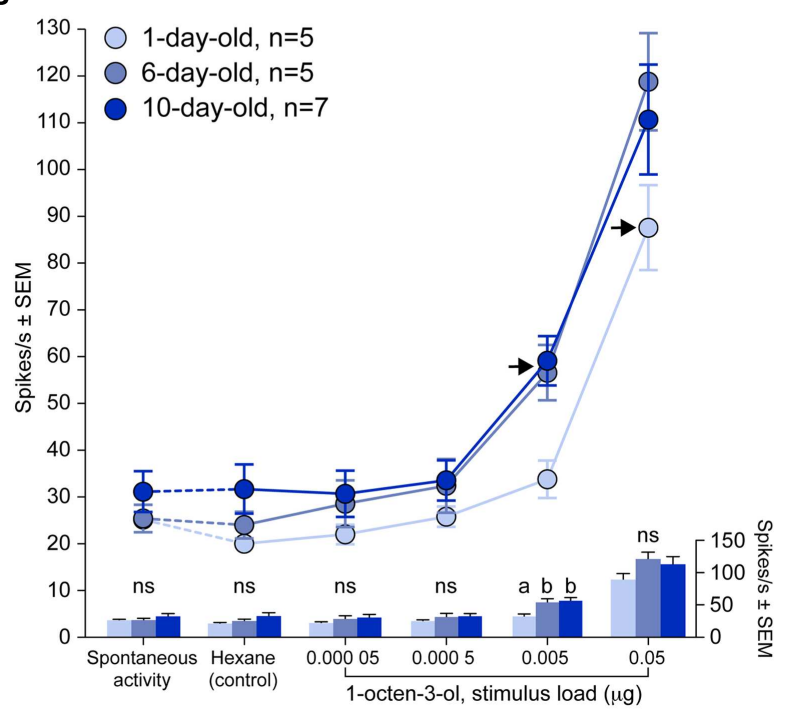

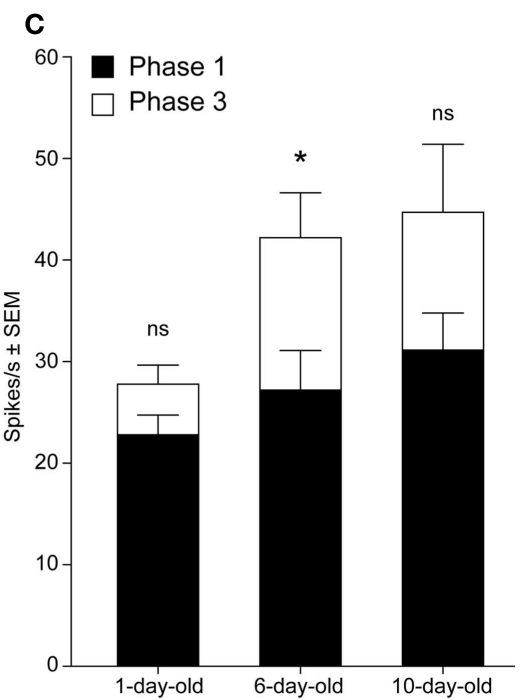

FIGURE 2 | Physiological development of the octenol receptor neuron. (A) Diagram outlining the methodology used to record action potentials of the " $\mathrm{C}$ " neuron in response to $1 \mathrm{~s}$ exposure of $\mathrm{CO}_{2}$-free air, hexane, or octenol (phase 2). A delay of $80 \mathrm{~ms}$ was observed between stimulation and the response. The use of $\mathrm{CO}_{2}$-free air allowed for the unobstructed observation of medium (inverted black triangle) and small-sized spikes, the latter being elicited by octenol. (B) Dose-dependent curves of mean octenol responses from the " $C$ " neuron of 1, 6, and 10 days old adult female mosquitoes. Points represented are mean \pm SEM $(n=5-7)$. Responses to octenol were compared to hexane control using a one-way ANOVA followed by Dunnett's post test $\left({ }^{* *} P<0.001 ;{ }^{*} P<0.01\right.$; ns, not significant). Detection threshold for 1 day old and $6 / 10$ days old insects was 0.05 and $0.005 \mu \mathrm{g}$, respectively (black arrows). The histograms below shows the comparison of the mean responses for each concentration (one-way ANOVA followed by Tukey's multiple comparison post test, $P<0.05$; ns, not significant). (C) The rate of spontaneous activity pre-stimulation (phase 1) and post-stimulation (phase 3) showed a significant change for 6 days old insects (Student $t$ test, ${ }^{*} P<0.05$; ns, not significant).

\section{RNA ISOLATION}

Five hundred and ten maxillary palps were dissected for each age group $(1,6$, and 10 days old animals). Each tissue collection was placed in dry ice and mechanically ground in TRIzol ${ }^{\circledR}$ (Invitrogen Life Technologies, Carlsbad, CA, USA). Total RNA was isolated following the manufacturer's protocol and sent to the Genomic Services Lab at Hudson Alpha Institute for Biotechnology (Huntsville, Alabama). Messenger RNA isolation and cDNA synthesis were prepared using the Illumi$\mathrm{na}^{\circledR}{ }^{\circledR}$ TruSeq $^{\mathrm{TM}}$ RNA Sample Preparation Kit (Illumina Inc., San Diego, CA, USA). Three libraries were sequenced and carried out on an Illumina HiSeq2000 to generate $50 \mathrm{bp}$ paired-end reads.

\section{POST-SEQUENCING ANALYSIS OF RNA-SEO DATA}

Reference genome and annotations for A. aegypti (AaegL1.3) were downloaded from VectorBase ${ }^{1}$. Output Fastq Illumina files were mapped to the reference genome with TopHat (Trapnell et al., 2009). Resulting sequence alignment files were uploaded into the Avadis NGS software (Strand Scientific Intelligence, CA, USA), where quantification and normalization were carried out. Prior to quantification using the Deseq normalization method, the read list was filtered to remove duplicate, single end, mate filtered, mate missing, one mate flip, both mate flip, and

\footnotetext{
${ }^{1}$ http://aaegypti.vectorbase.org/GetData/Downloads/
} 
unaligned reads. Transcript expression levels for AaOr8 (gene accession number: AAEL012254), AaOr49 (AAEL001303), AaOrco (AAEL005776), AaGr1 (AAEL002380), AaGr2 (AAEL002167), and AaGr3 (AAEL010058) were reported in units of Reads Per Kilo-base per Million reads mapped (RPKMs).

\section{SENSORY PHYSIOLOGY}

Single-cell electrophysiological recordings were carried out on the basiconic sensilla of the maxillary palp of 1,6 , and 10 days old female A. aegypti as previously described (Grant and Dickens, 2011). Mosquitoes were immobilized by brief chilling at $-20^{\circ} \mathrm{C}$ and glued on their side to a mounting stage in order to position the basiconic sensilla on the maxillary palp for access by the recording electrode. Both the recording and reference tungsten electrodes were electrolytically sharpened to a tip of less than $1 \mu \mathrm{m}$ diameter. The recording electrode was placed at the base of a basiconic sensillum while the reference electrode was inserted into the insect's compound eye. Serial dilutions of racemic octenol (>98\%, Fluka Chemical Corp., Milwaukee, WI, USA) were prepared in spectrophotometric grade hexane. Volatiles emanating from $5 \mu \mathrm{L}$ aliquots of the serial dilutions applied to filter paper strips were carried over the preparation by compressed air (Ultra Zero Grade, $>0.5 \mathrm{ppm} \mathrm{CO} 2,665 \mathrm{~mL} / \mathrm{min}$ ). Stimulus duration was $1 \mathrm{~s}$. The analog signal was amplified and filtered (bandpass 300$1000 \mathrm{~Hz}$ ) using a preamplifier (model P15D, Grass Instrument Corp., Quincy, MA, USA). Further amplification and signal digitization were performed by an IDAC 4 (Syntech, Kirchzarten, Germany). Data were visualized, recorded, and analyzed using AutoSpike (Syntech, Kirchzarten, Germany) software and a microcomputer. The smallest amplitude spikes were invariably elicited by octenol. Spikes were counted for three time periods: $1 \mathrm{~s}$ prior to stimulus onset (Phase 1), the 1 s stimulus duration (Phase 2), and 1 s following the stimulus (Phase 3; Figure 2A).

\section{BIOASSAY}

Behavioral bioassays of single mosquitoes were conducted using a Plexiglas ${ }^{\circledR}$ dual-choice olfactometer (custom made by Precision Plastics Inc., Beltsville, MD, USA, Figure 3) designed to measure upwind attraction, modified after Geier et al. (1999) and Bosch et al. (2000). The most upwind section of the olfactometer consisted of two $12.7 \mathrm{~cm}$ lengths plastic cylinders (diameter $10.2 \mathrm{~cm}$ ) containing aluminum tubes (diameter $2 \mathrm{~cm}$, length $30 \mathrm{~cm}$ ). A laminar flow was made by passing the airflow through a spongy steel insert within the tubes. Air flowed through each of the two cylinders, through mesh screens (to prevent mosquito entry), and into the cylinders (inner diameter $10.2 \mathrm{~cm}, 12.7 \mathrm{~cm}$ long) corresponding to the control chamber and the test chamber (in which volatile stimuli were introduced). Each chamber contained a sliding screen door which could be closed at the conclusion of each experiment. The two chambers were attached to a rectangular box $(10.8 \mathrm{~cm} \mathrm{H} \times 30.5 \mathrm{~cm} \mathrm{~W} \times 17.1 \mathrm{~cm} \mathrm{~L})$ opposite a single cylinder $(10.2 \mathrm{~cm}$ inner diameter, $30.5 \mathrm{~cm}$ long) where mosquitoes were individually released from cages. Release cages were cylinders $(10.2 \mathrm{~cm}$ inner diameter, $11.4 \mathrm{~cm}$ long) with screen covering one end and a sliding door with screen on the other end. All parts were designed to be easily removable prior to cleaning with distilled water and Alconox ${ }^{\circledR}$ laboratory detergent. Experiments were conducted under red light conditions using four Safelight lamps (Kodak, USA) equipped with Roscolux medium red filters (Roscolab Ltd., London, UK) mounted $35.6 \mathrm{~cm}$ above the olfactometer. Intensity and wavelength of emitted light was measured with a spectrometer (USB4000, Ocean Optics, Dunedin, FL, USA).

Air supplied by a pump located outside of the bioassay room (Gast, MI, USA) was filtered with a ultra-high capacity hydrocarbon trap (Restek, PA, USA) and humidified by passing through deionized water. The airstream was maintained at $27^{\circ} \mathrm{C}$ and $60-73 \%$ relative humidity as measured by a hygrothermometer (EA80, Extech Instruments, Waltham, MA, USA). The flow rate was $6 \mathrm{~L} / \mathrm{min}(30 \pm 15 \mathrm{~cm} / \mathrm{s}$ airspeed as measured by a hot-wire anemometer, model 441S, Kurtz, CA, USA; accuracy $\pm 0.01 \mathrm{~m} / \mathrm{s}$ ).

Custom air comprised of $4.5 \% \mathrm{CO}_{2}$ (AirGas Inc., NH, USA) was regulated by a flowmeter (model N082-03 Cole-Parmer, IL, USA) at $1.6 \mathrm{~L} / \mathrm{min}$. A stimulus controller (type CS-55, Syntech, The Netherlands) was programmed for $2 \mathrm{~s}$ puffs at $2 \mathrm{~s}$ intervals to simulate discontinuous human breath. Puffs of $\mathrm{CO}_{2}$ were injected into the continuous air flow through a curved Pasteur pipette inserted through a hole in the aluminum tube carrying the airstream in test chamber.

Octenol was diluted in hexane to make a $10 \mu \mathrm{g} / \mu \mathrm{L}$ solution as in the physiological studies. Ten microliters of this solution was applied on a filter paper strip (size $1 \mathrm{~cm} \times 4.5 \mathrm{~cm}$, Whatman No. 1) to give $100 \mu \mathrm{g}$ of 1 -octen-3-ol on the paper. The hexane solvent was allowed to evaporate under a hood for approximately $20 \mathrm{~s}$ at room temperature prior to placing the filter paper in the aluminum tube of the test chamber.

\section{BEHAVIORAL TEST}

Non-blood-fed female mosquitoes were aspirated individually into release cages $20 \mathrm{~min}$ prior to the experiment. Mosquitoes were acclimated for $10 \mathrm{~min}$ under normal light then $10 \mathrm{~min}$ under red light. The test was started by slowly opening the release cage. Each mosquito was observed for $10 \mathrm{~min}$ for the following behaviors: activation (mosquito took flight and left the release chamber) and attraction (mosquito entered the control or test chamber). The insect was then removed from the olfactometer and odorant-free air flowed through the device to remove any contaminant for $5 \mathrm{~min}$ prior to the next experiment. Between each test, the control and test chambers were inverted to avoid any preference for the left or the right arm of the olfactometer. $\mathrm{CO}_{2}$ alone and $\mathrm{CO}_{2}+$ octenol were alternated and 20 individuals were tested for each treatment. Tests were conducted from 10:00 to 17:00 h during the light cycle under which the insects were reared.

\section{BOOTSTRAP ANALYSIS}

The observed data, 20 mosquitoes for each of the three ages $\times$ two treatment groups, although time consuming and labor intensive to bioassay, were inadequate sample sizes to produce estimates of the proportion of mosquitoes attracted with sufficient accuracy and precision to statistically detect differences of practical biological importance. Therefore, the original observed data set, was considered to be one replicate of the experiment, and was resampled to acquire bootstrap (Manly, 2007) replicates of the experiment. Estimates of the proportion of mosquitoes attracted to a stimulus were obtained by fitting an over-dispersed Binomial model using 
the glmer function in the lme 4 package in the $R^{2}$ statistical freeware environment. The over-dispersed Binomial ANOVA model was fit to the observed data, then to the observed data plus the first resampled replicate (i.e., two full replicates of the experiment). Each time another resampled replicate was added to the data set, the ANOVA model was fit again; allowing identification of a necessary and sufficient amount of replication for statistical detection of biologically and practically important differences (when they exist) in proportion of mosquitoes attracted.

\section{RESULTS \\ ELECTROPHYSIOLOGICAL RESPONSE OF OCTENOL-SENSITIVE NEURONS}

We recorded the electrical activity of the " $\mathrm{C}$ " neuron (smallest amplitude spike) using the single-cell recording technique in 1, 6, and 10 days old adult female $A$. aegypti mosquitoes. The activity of the "C" neuron was divided into three $1 \mathrm{~s}$ periods: before stimulation (phase 1), during stimulation (phase 2), and after stimulation (phase 3; Figure 2A).

The firing rate of the " $\mathrm{C}$ " neuron elicited by hexane and increasing stimulus loads of racemic 1-octen-3-ol were analyzed during phase 2 (Figure 2B). The firing rate evoked by hexane did not differ from the rate of the spontaneous activity, and the three age groups did not differ in their rate of spontaneous activity (oneway ANOVA, $P=0.4044$; Figure 2B). The detection threshold was determined by comparing responses elicited by serial dilutions of octenol to the hexane control. Responses to $0.05 \mu \mathrm{g}$ octenol was the lowest dose statistically different from hexane in 1 day old animals. The detection threshold for 6 and 10 days old animal was 10 times lower at $0.005 \mu \mathrm{g}$ octenol (Figure 2B). While the detection threshold was clearly established for the three age groups, we did not detect a significant difference with the highest stimulus load (Figure 2B).

${ }^{2} \mathrm{http}$ //www.r-project.org
The physiological recovery of the " $\mathrm{C}$ " neuron was assessed by comparing the rate of the spontaneous activity $1 \mathrm{~s}$ before (phase 1) and $1 \mathrm{~s}$ after (phase 3) stimulation (Figure 2A). Spontaneous activity showed a statistically different (Student $t$ test, $P<0.05$ ) recovery rate in 6 days old animals at the highest concentration $(0.05 \mu \mathrm{g}$; Figure 2C). For all other concentrations and for all other age groups the rate of spontaneous activity always recovered during phase 3 (data not shown).

\section{DEVELOPMENT OF BEHAVIOR: ACTIVATION AND ATTRACTION}

Except during mating, when adult males form occasional swarms to attract females (Goeldi, 1905; Hartberg, 1971; Cabrera and Jaffe, 2007), mosquitoes have a solitary lifestyle. Thus, individual females were tested in our dual-choice bioassay (Figure 3). Carbon dioxide, a known activator of upwind flight, was used alone or in the presence of octenol. We looked at female mosquitoes distributed in three age groups for a total of six experiments. One day old animals were not attracted to either $\mathrm{CO}_{2}$ alone or $\mathrm{CO}_{2}+$ octenol (Figure 4). While $\mathrm{CO}_{2}$ alone and $\mathrm{CO}_{2}+$ octenol elicited strong attraction from 6 and 10 days old animals, we could not detect any difference between the two stimuli and the two age groups.

Simple ANOVA analysis did not identify a significant difference between the two stimuli at either of the older age groups. Since the nature of the bioassay of individual mosquitoes was time consuming and labor intensive, additional replicate observations were impractical. Therefore, we adopted a simulation-based approach to generate bootstrap samples. The observed data, one replicate of the experiment, was resampled to acquire bootstrap (Manly, 2007) replicates of the experiment, to determine potential outcomes of additional sampling. The "six replicate" result, the observed data plus five resampled replicates, was the smallest number of replicates to show a reduced attraction to both treatments in 10 days old insects ( $P<0.001$; data not shown). The "12 replicate" bootstrap result indicated that $\mathrm{CO}_{2}+$ octenol was more attractive than $\mathrm{CO}_{2}$ alone in 6 days old animals $(P<0.01$; Figure 4).

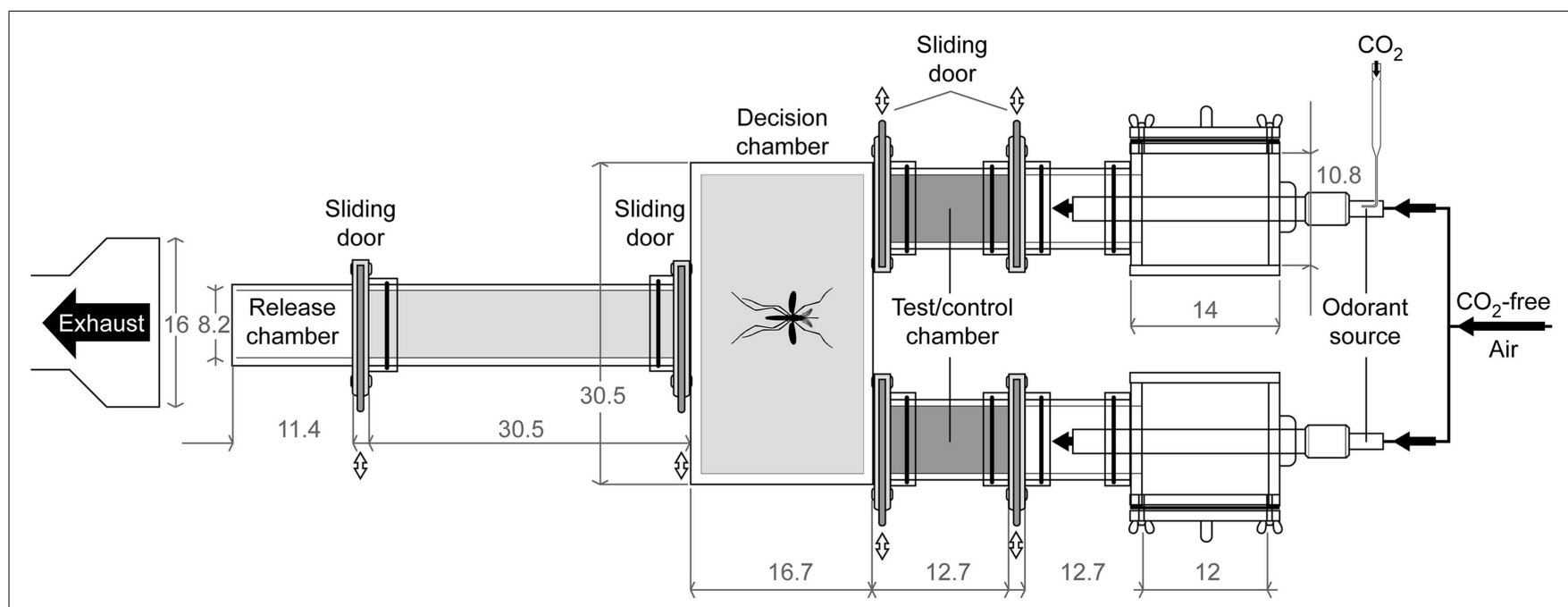

FIGURE 3 | Technical 2D-diagram of the dual-choice bioassay. Mosquitoes trapped in the area outside the release and test/control chambers (light gray area) were counted as activated. Mosquitoes that have flown to the test/control chambers (dark gray areas) were counted as attracted. Dimensions given in cm. 


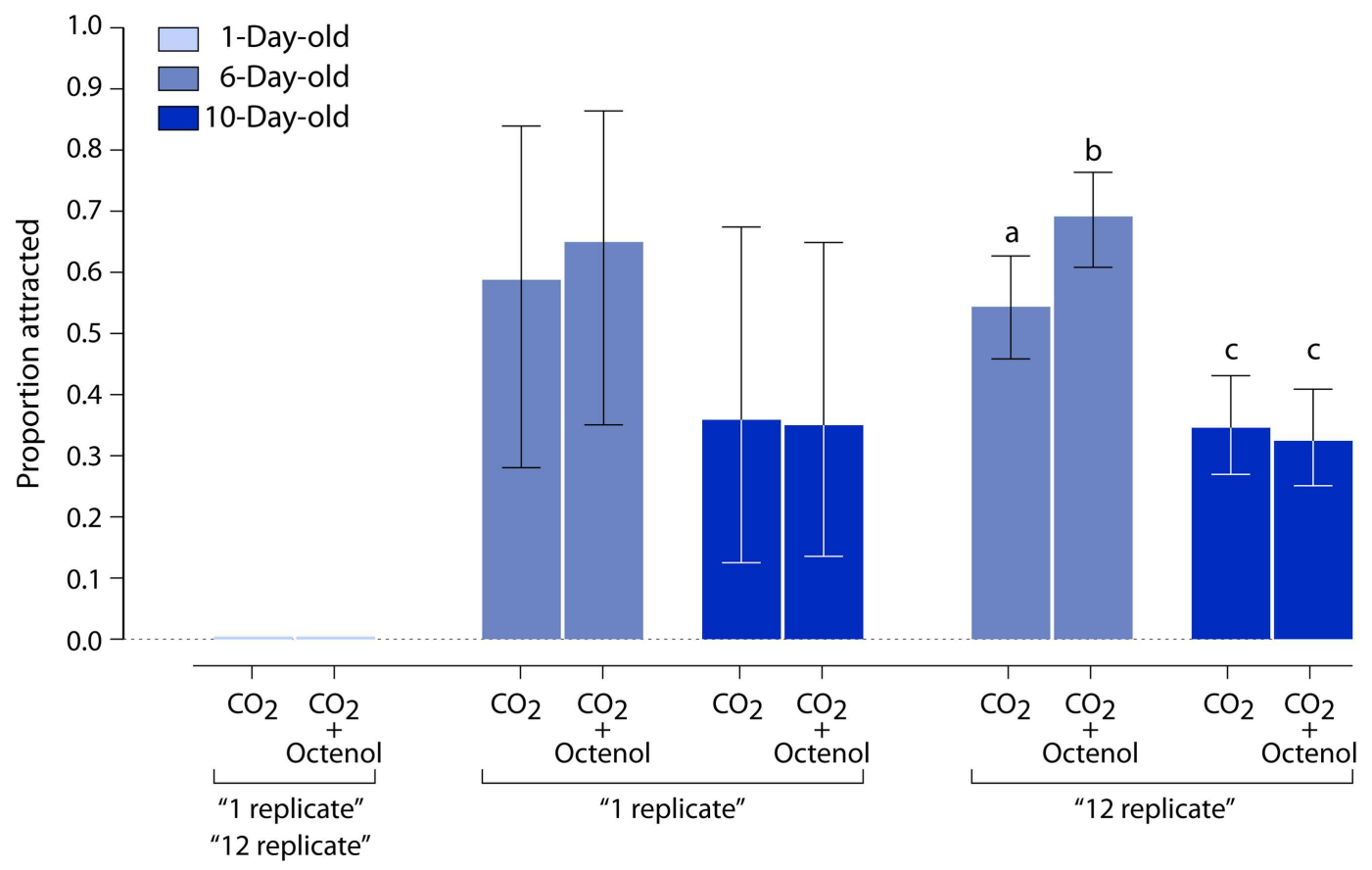

FIGURE 4 | Influence of age on behavioral responses to $\mathrm{CO}_{2}$ and octenol. Newly emerged female mosquitoes ( 1 day old) are neither activated by $\mathrm{CO}_{2}$ nor attracted to $\mathrm{CO}_{2}+$ octenol. Six day old individuals show significant attraction to both $\mathrm{CO}_{2}$ and $\mathrm{CO}_{2}+$ octenol. Ten day old animals exhibit lower $\mathrm{CO}_{2}$ activation and no attraction to $\mathrm{CO}_{2}+$ octenol. Vertical bars represent $95 \%$ confidence intervals.

\section{PROFILING CHEMOSENSORY GENE EXPRESSION}

High-throughput sequencing (RNA-seq) was used to identify and measure the abundance of expressed genes in the maxillary palps of 1,6 , and 10 days old female mosquitoes. Among the thousands of genes present in each sample, we chose to focus on chemosensory receptor genes known to be expressed in the palps including AaOr8, AaOr49, AaOrco, AaGr1, AaGr2, and AaGr3. AaOr8-Orco, $A a G r 1$, and $A a G r 3$ are presumably expressed in the basiconic sensilla based on their function in octenol (Bohbot and Dickens, 2009) and $\mathrm{CO}_{2}$ reception (Erdelyan et al., 2011), respectively. All six genes exhibited the same general trend: robust expression at emergence with increased expression occurring between 1 and 6 days old animals before some leveling off in 10 days old insects (Figure 5).

\section{DISCUSSION}

Single-cell recordings and molecular expression data indicate that at emergence, octenol-sensitive neurons are physiologically competent, but have not reached maturity. We have confirmed that AaOr8 and AaOrco expression levels are significant in newly emerged mosquitoes (Bohbot et al., 2007) and continue to increase after adult emergence. Absolute RPKM (estimation of gene expression) values were consistent with previously described levels of transcripts in the antennae of A. gambiae (Pitts et al., 2011), confirming that these genes are highly expressed in the maxillary palps of $A$. aegypti. Although we did not report evidence that the encoded proteins are present in the dendritic processes of the octenol ORNs, based on the observed physiological responses, we speculate that protein translation reflects RNA expression levels.
The same observation can be made for $A a G r 1$ and $A a G r 3\left(\mathrm{CO}_{2}\right.$ receptor), as well as for $A a G r 2$ and AaOr49. A previous study showed that $A a G r 1,2,3$ expression begins during the pupal stage and continues into adulthood (Erdelyan et al., 2011), which is consistent with our gene expression analysis.

Maturation of neuronal responsiveness to octenol resembles the developmental pattern of the $\mathrm{CO}_{2}$ response (Grant and O'Connell, 2007) in female $A$. aegypti. This similarity may be a result of common morphological and hormonal factors in sense organ development (Clements, 1999) as both neurons occupy the same basiconic sensilla on the palps (McIver, 1972). According to Clements (1999), the first $24 \mathrm{~h}$ following emergence is a vulnerable period for female $A$. aegypti whose various parts of the body, including the mouthparts, are undergoing hardening or sclerotization. Coincidently, these young insects exhibit reduced physiological competency, do not show any behavioral attraction to a human hand, and do not blood-feed. Davis (1984) showed that the sensitivity of lactic-acid sensitive neurons develops with age and is also dependent on the blood-feeding status of female $A$. aegypti. Juvenile hormone released by the corpora allata has been implicated in regulating biting behavior in newly emerged Culex mosquitoes (Meola and Petralia, 1980) and might play a role in A. aegypti (Klowden and Blackmer, 1987). This would indicate that a neural or humoral mechanism is preventing newly emerged female mosquitoes from feeding.

Could a similar mechanism govern cuticle hardening and neural maturation? Both the molecular and physiological data reported here indicate that the peripheral olfactory system has reached sufficient maturity to provide sensory input for eliciting 


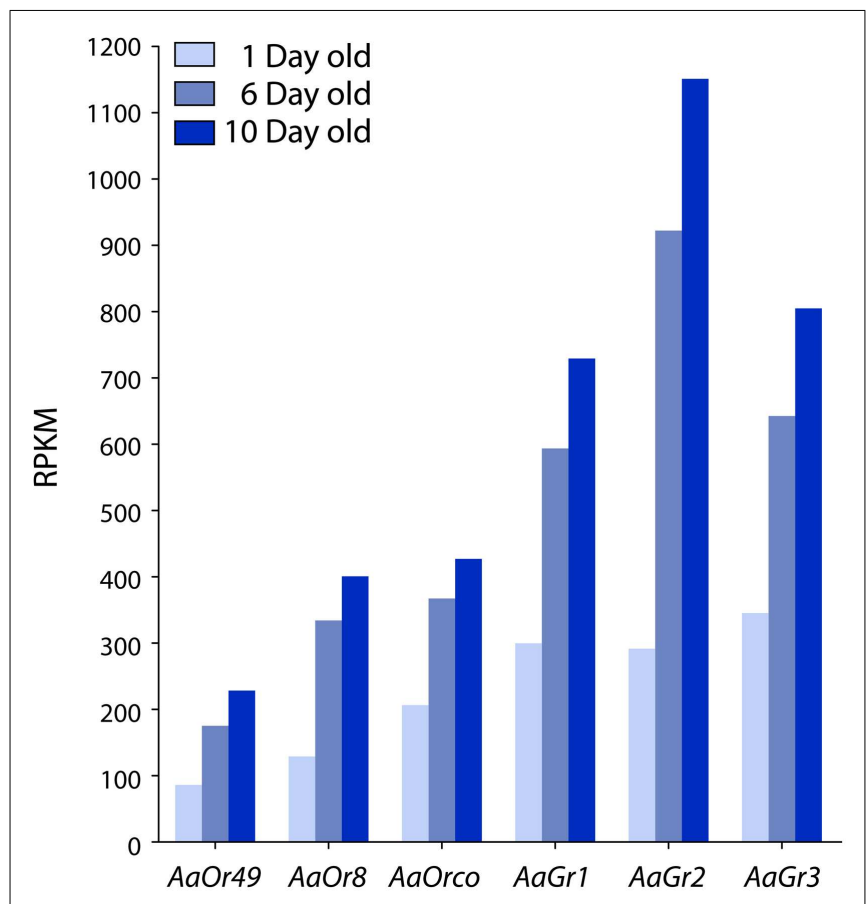

FIGURE 5 | Temporal expression of $\mathrm{AaOr}$ and $\mathrm{AaGr}$ genes in the maxillary palps of female Aedes aegypti defined by RNA-seq. All genes were highly expressed throughout adulthood. The largest increase in expression occurs between 1 and 6 days old animals, and is followed by a plateau in 10 days old mosquitoes.

behavior. However, behavioral responses in the form of attraction to $\mathrm{CO}_{2}$ or $\mathrm{CO}_{2}+$ octenol were absent, which indicates a deficit of responsive central neural pathways or a humoral control mechanism of these pathways. Presynaptic and postsynaptic modulation of olfactory processing (Wang, 2011) have been reported in multiple insect species and involve biogenic amines (Kuppers and Thürm, 1975; Linn and Roelofs, 1986; Pophof, 2000, 2002; Grosmaitre et al., 2001; Spivak et al., 2003; Zhukovskaya and Kapitsky, 2006; Flecke and Stengl, 2009; Vergoz et al., 2009; McQuillan et al., 2012), dopamine (Andersen et al., 2006), tachykinins (Winther

\section{REFERENCES}

Andersen, J. P., Schwartz, A., Gramsbergen, J. B., and Loeschcke, V. (2006). Dopamine levels in the mosquito Aedes aegypti during adult development, following blood feeding and in response to heat stress. J. Insect Physiol. 52, 1163-1170.

Bohbot, J., Pitts, R. J., Kwon, H. W., Rutzler, M., Robertson, H. M., and Zwiebel, L. J. (2007). Molecular characterization of the Aedes aegypti odorant receptor gene family. Insect Mol. Biol. 16, 525-537.

Bohbot, J. D., and Dickens, J. C. (2009). Characterization of an enantioselective odorant receptor in the yellow fever mosquito Aedes aegypti. PLoS ONE 4:e7032. doi:10.1371/journal.pone.0007032
Bosch, O. J., Geier, M., and Boeckh, J. (2000). Contribution of fatty acids to olfactory host finding of female Aedes aegypti. Chem. Senses 25, 323-330.

Cabrera, M., and Jaffe, K. (2007). An aggregation pheromone modulates lekking behavior in the vector mosquito Aedes aegypti (Diptera: culicidae). J. Am. Mosq. Control Assoc. 23, $1-10$.

Clements, A. N. (1999). The Biology of Mosquitoes. New York: CABI Publishing.

Davis, E. E. (1984). Development of lactic acid-receptor sensitivity and host-seeking behaviour in newly emerged female Aedes aegypti mosquitoes. J. Insect Physiol. 30, 211-215.

et al., 2006; Ignell et al., 2009), and short neuropeptide F (Root et al., 2011), as well as insulin (Root et al., 2011).

The activation and attraction elicited by $\mathrm{CO}_{2}$ and $\mathrm{CO}_{2}+$ octenol in 6 days old animals, and the following reduction of the same behaviors associated with 10 days old animals, despite full peripheral olfactory competency, suggest central nervous system modulation by some humoral factor. Another possibility is that senescence, defined by a post-maturation decline in cell integrity and function, may be the cause of reduced activation and attraction in older insects (Seabrook et al., 1979, 1987; Fescemyer and Hanson, 1990).

In conclusion, our results, taken together with previous investigations, show that the physiological development of octenol, lactic acid, and $\mathrm{CO}_{2}$-sensitive receptor neurons are synchronous and age-dependent in adult mosquitoes. Our data indicate concerted changes in chemosensory gene expression that correlate with increased olfactory sensitivity between 1 and 6 days old insects. We speculate that for older animals, age leads to an inevitable decline in sensory function as shown for other insects (Seabrook et al., 1979; Dickens and Moorman, 1990). As octenol, $\mathrm{CO}_{2}$, lactic acid, and other chemicals are vital chemical cues for the ecological success of mosquitoes (Takken and Knols, 1999), it is important to understand which of these sensory modalities may provide the most stable targets for chemical disruption.

\section{ACKNOWLEDGMENTS}

We wish to thank the following individuals: Dr. Shawn E. Levy and Nripesh Prasad at the Genomic Services Lab at HudsonAlpha Institute for Biotechnology for Illumina sequencing and data analyses; Dr. Gary Bauchan (The Electron and Confocal Microscope Unit, USDA, ARS) for his assistance in obtaining scanned electron microscope images of $A$. aegypti. We are also grateful to Drs. Richard Vogt and Jackson Sparks (University of South Carolina) whose useful comments have improved the quality of the manuscript. This work was supported in part by a grant to Joseph C. Dickens from the Deployed War Fighter Protection (DWFP) Research Program funded by the Department of Defense through the Armed Forces Pest Management Board (AFPMB).

Dickens, J. C., and Moorman, E. E. (1990). Maturation and maintenance of electroantennogram responses to pheromone and host odors in boll weevils, Anthonomus grandis boh. (Col., Curculionidae) fed their host plant or artificial diet. J. Appl. Entomol. 109, 470-480.

Erdelyan, C. N., Mahood, T. H., Bader, T. S., and Whyard, S. (2011). Functional validation of the carbon dioxide receptor genes in Aedes aegypti mosquitoes using RNA interference. Insect Mol. Biol. 21, 119-127.

Fescemyer, H. W., and Hanson, F. E. (1990). Male European corn borer, Ostrinianubilalis (hubner), antennal responses to analogs of its sex pheromone. J. Chem. Ecol. 16, 773-790.
Flecke, C., and Stengl, M. (2009). Octopamine and tyramine modulate pheromone-sensitive olfactory sensilla of the hawkmoth Manduca sexta in a time-dependent manner. J. Comp. Physiol. A Neuroethol. Sens. Neural Behav. Physiol. 195, 529-545.

Galizia, C. G., and Rossler, W. (2010). Parallel olfactory systems in insects: anatomy and function. Annu. Rev. Entomol. 55, 399-420.

Geier, M., Bosch, O. J., and Boeckh, J. (1999). Ammonia as an attractive component of host odour for the yellow fever mosquito, Aedes aegypti. Chem. Senses 24, 647-653.

Goeldi, E. A. (1905). Os mosquitos no Para. Mem. Museu E. Goeldi (Belem) 4, 1-154. 
Grant, A., and O'Connell, R. (1996). "Electrophysiological responses from receptor neurons in mosquito maxillary palp sensilla," in Olfaction in Mosquito-Host Interactions, eds G. R. Block and G. Cardew (Chichester: Wiley Press), 233-253.

Grant, A. J., and Dickens, J. C. (2011). Functional characterization of the octenol receptor neuron on the maxillary palps of the yellow fever mosquito, Aedes aegypti. PLoS ONE 6:e21785. doi:10.1371/journal.pone.0021785

Grant, A. J., and O'Connell, R. J. (2007). Age-related changes in female mosquito carbon dioxide detection. J. Med. Entomol. 44, 617-623.

Grosmaitre, X., Marion-Poll, F., and Renou, M. (2001). Biogenic amines modulate olfactory receptor neurons firing activity in Mamestra brassicae. Chem. Senses 26, 653-661.

Hansson, B. S., and Stensmyr, M. C. (2011). Evolution of insect olfaction. Neuron 72, 698-711.

Hartberg, W. K. (1971). Observations on the mating behavior of Aedes aegypti in nature. Bull. World Health Organ. 45, 847-850.

Ignell, R., Root, C. M., Birse, R. T., Wang, J. W., Nassel, D. R., and Winther, A. M. (2009). Presynaptic peptidergic modulation of olfactory receptor neurons in Drosophila. Proc. Natl. Acad. Sci. U.S.A. 106, 13070-13075.

Jones, W. D., Cayirlioglu, P., Kadow, I. G., and Vosshall, L. B. (2007). Two chemosensory receptors together mediate carbon dioxide detection in Drosophila. Nature 445, 86-90.

Jones, W. D., Nguyen, T. A., Kloss, B., Lee, K. J., and Vosshall, L. B. (2005). Functional conservation of an insect odorant receptor gene across 250 million years of evolution. Curr. Biol. 15, R119-R121.

Kent, L. B., Walden, K. K., and Robertson, H. M. (2008). The Gr family of candidate gustatory and olfactory receptors in the yellow-fever mosquito Aedes aegypti. Chem. Senses 33, 79-93.

Klowden, M. J., and Blackmer, J. L. (1987). Humoral control of preoviposition behaviour in the mosquito, Aedes aegypti. J. Insect Physiol. 33, 689-692.

Kuppers, J., and Thürm, U. (1975). Humoral control of an ion transport mechanism at epithelial receptors of insects (in German). Verh. Dtsch. Zool. Ges. 1974, 46-50.

Linn, C. E., and Roelofs, W. L. (1986). Modulatory effects of octopamine and serotonin on male sensitivity and periodicity of response to sex pheromone in the cabbage looper moth, Trichoplusia ni. Arch. Insect Biochem. Physiol. 3, 161-171.

Lu, T., Qiu, Y. T., Wang, G., Kwon, J. Y., Rutzler, M., Kwon, H. W., et al. (2007). Odor coding in the maxillary palp of the malaria vector mosquito Anopheles gambiae. Curr. Biol. 17, 1533-1544.

Manly, B. F. J. (2007). Randomization, Bootstrap and Monte Carlo Methods in Biology, 3rd Edn. Boca Raton, FL: CRC Press.

McIver, S. B. (1972). Fine structure of pegs on the palps of female culicine mosquitoes. Can. J. Zool. 50, 571-576.

McIver, S. B. (1982). Review article: sensilla of mosquitoes (Diptera: culicidae). J. Med. Entomol. 19, 489-535.

McQuillan, H. J., Barron, A. B., and Mercer, A. R. (2012). Ageand behaviour-related changes in the expression of biogenic amine receptor genes in the antennae of honey bees (Apis mellifera). J. Comp. Physiol. A Neuroethol. Sens. Neural. Behav. Physiol. 198, 753-761.

Meola, R. W., and Petralia, R. S. (1980). Juvenile hormone induction of biting behavior in Culex mosquitoes. Science 209, 1548-1550.

Pitts, R. J., Rinker, D. C., Jones, P. L., Rokas, A., and Zwiebel, L. J. (2011). Transcriptome profiling of chemosensory appendages in the malaria vector Anopheles gambiae reveals tissue- and sex-specific signatures of odor coding. BMC Genomics 12:271. doi:10.1186/1471-2164-12271

Pophof, B. (2000). Octopamine modulates the sensitivity of silkmoth pheromone receptor neurons. J. Comp. Physiol. A 186, 307-313.

Pophof, B. (2002). Octopamine enhances moth olfactory responses to pheromones, but not those to general odorants. J. Comp. Physiol. A Neuroethol. Sens. Neural. Behav. Physiol. 188, 659-662.

Robertson, H. M., and Kent, L. B. (2009). Evolution of the gene lineage encoding the carbon dioxide receptor in insects. J. Insect Sci. 9, 19.

Root, C. M., Ko, K. I., Jafari, A., and Wang, J. W. (2011). Presynaptic facilitation by neuropeptide signaling mediates odor-driven food search. Cell 145, 133-144.

Seabrook, W. D., Hirai, K., Shorey, H. H., and Gaston, L. K. (1979). Maturation and senescence of an insect chemosensory response. J. Chem. Ecol. 5, 587-594.

Seabrook, W. D., Lin, C. E., Dyer, L. J., and Shorey, H. H. (1987). Comparison of electroantennograms from female and male cabbage looper moths (Trichoplusia ni) of different ages and for various pheromone concentrations. J. Chem. Ecol. 13, 1443-1453.

Spivak, M., Masterman, R., Ross, R. and Mesce, K. A. (2003). Hygienic behavior in the honey bee (Apis mellifera L.) and the modulatory role of octopamine. J. Neurobiol. 55, 341-354.

Takken, W., and Kline, D. L. (1989). Carbon dioxide and 1-octen-3-ol as mosquito attractants. J. Am. Mosq. Control Assoc. 5, 311-316.

Takken, W., and Knols, B. G. (1999). Odor-mediated behavior of Afrotropical malaria mosquitoes. Annu. Rev. Entomol. 44, 131-157.
Trapnell, C., Pachter, L., and Salzberg, S. L. (2009). TopHat: discovering splice junctions with RNA-Seq. Bioinformatics 25, 1105-1111.

Vergoz, V., McQuillan, H. J., Geddes, L. H., Pullar, K., Nicholson, B. J., Paulin, M. G., et al. (2009). Peripheral modulation of worker bee responses to queen mandibular pheromone. Proc. Natl. Acad. Sci. U.S.A. 106, 20930-20935.

Wang, J. W. (2011). Presynaptic modulation of early olfactory processing in Drosophila. Dev. Neurobiol. 72, 87-99.

Winther, A. M., Acebes, A., and Ferrus, A. (2006). Tachykinin-related peptides modulate odor perception and locomotor activity in Drosophila. Mol. Cell. Neurosci. 31, 399-406.

Zhukovskaya, M. I., and Kapitsky, S. V. (2006). Activity modulation in cockroach sensillum: the role of octopamine. J. Insect Physiol. 52, 76-86.

Conflict of Interest Statement: The authors declare that the research was conducted in the absence of any commercial or financial relationships that could be construed as a potential conflict of interest.

Received: 28 December 2012; accepted: 14 February 2013; published online: 07 March 2013.

Citation: Bohbot JD, Durand NF, Vinyard BT and Dickens JC (2013) Functional development of the octenol response in Aedes aegypti. Front. Physiol. 4:39. doi:10.3389/fphys.2013.00039

This article was submitted to Frontiers in Invertebrate Physiology, a specialty of Frontiers in Physiology.

Copyright (c) 2013 Bohbot, Durand, Vinyard and Dickens. This is an openaccess article distributed under the terms of the Creative Commons Attribution License, which permits use, distribution and reproduction in other forums, provided the original authors and source are credited and subject to any copyright notices concerning any third-party graphics etc. 